Pacific Journal of Mathematics

A DUALITY BETWEEN TRANSPOTENCE ELEMENTS AND 


\section{A DUALITY BETWEEN TRANSPOTENCE ELEMENTS AND MASSEY PRODUCTS}

\section{Byron Drachman and David Kraines}

The purpose of this note is to show that if $v$ is an element whose suspension is nonzero, and if $u$ is dual to $v$, then the transpotence $\varphi_{k}(v)$ is defined and nonzero if and only if the $k$-Massey product $\langle u\rangle^{k}$ is defined and nonzero.

We wish to thank Dr. Samuel Gitler for a helpful conversation on this material.

\section{Preliminaries.}

1.1. The Cobar Construction: (Adams [1]). Let $C$ be a simply connected $D G A$ coalgebra over $K$ with co-associative diagonal map where $K$ is a commutative ring with unit. The Cobar Construction $\vec{F}(C)$ is the direct sum of the $n$-fold tensor products of the desuspension of $\bar{C}=\operatorname{Ker}(\varepsilon)$ where $\varepsilon: C \rightarrow K$ is the augmentation. Suppose $C$ has a differential $\left\{d_{n}: C_{n} \rightarrow C_{n-1}\right\}$. A typical element is a linear combination of elements of the form

$$
x=s^{-1}\left(c_{1}\right) \otimes \cdots \otimes s^{-1}\left(c_{n}\right)=\left[c_{1}|\cdots| c_{n}\right]
$$

where $x$ has bidegree $(-n, m)$ and $m=\sum_{i=1}^{n}$ degree $\left(c_{i}\right)$. The differential in $\bar{F}(C)$ is defined on elements of bidegree $\left(-1,{ }^{*}\right)$ by

$$
d[c]=[-d c]+\sum_{i}(-1)^{\operatorname{deg} c_{i}{ }^{\prime}}\left[c_{i}{ }^{\prime} \mid c_{i}^{\prime \prime}\right]
$$

where

$$
\Delta(c)=c \otimes 1+1 \otimes c+\sum_{i} c_{i}{ }^{\prime} \otimes c_{i}{ }^{\prime \prime}
$$

$\Delta: C \rightarrow C \otimes C$ being the diagonal mapping of $C$. The differential is extended to all of $\vec{F}(C)$ by the requirement that $\vec{F}(C)$ be a $D G A$ algebra.

If $C$ has a differential of degree +1 instead of -1 , we no longer ask that $C$ be a simply connected but only connected, and the element $\left[c_{1}|\cdots| c_{n}\right]$ is assigned bidegree $(n, m)$.

1.2. The Bar Construction. Let $A$ be a connected associative $D G A$ algebra over $K$. Let $\varepsilon: A \rightarrow K$ be the augmentation. Let $\bar{A}=\operatorname{ker} \varepsilon$. Then the Bar Construction $\bar{B}(A)$ is the direct sum of the $n$-fold tensor products of the suspension of $\bar{A}$. Let

$$
\left\{d_{n}: A_{n} \rightarrow A_{n-1}\right\}
$$


be the differential in $A . \bar{B}(A)$ is bigraded by assigning the element $\left[a_{1}|\cdots| a_{n}\right]$ degree $(n, m)$ where $m=\sum_{i=1}^{n} \operatorname{deg} a_{i} \cdot \quad \bar{B}(A)$ has a differential $d=d_{E}+d_{I}$ where

$$
\begin{aligned}
d_{E}\left(\left[a_{1}|\cdots| a_{n}\right]\right) & =\sum_{i=1}^{n-1}(-1)^{u(i)}\left[a_{1}|\cdots| a_{i} a_{i+1}|\cdots| a_{n}\right] \\
d_{I}\left(\left[a_{1}|\cdots| a_{n}\right]\right) & =\sum_{i=1}^{n}(-1)^{u(i-1)}\left[a_{1}|\cdots| \partial a_{i}|\cdots| a_{n}\right]
\end{aligned}
$$

where

$$
u(i)=i+\sum_{k=1}^{i} \operatorname{deg} a_{k} .
$$

We also mention that $[a|\cdots(k) \cdots| a]$ is $\gamma_{k}[a]$, the $k$ th divided power of $[a]$.

If instead of the above the differential of $A$ has degree +1 , we put the bidegree of $\left[a_{1}|\cdots| a_{n}\right]$ to be $(-n, m)$. In this case we will always assume $A$ is simply connected.

1.3. The Suspension Map. In the case of the Bar Construction the suspension map $\sigma: H_{*}(A) \rightarrow H_{*}(\bar{B}(A))$ is represented by $a \rightarrow[a]$. In the case of the Cobar Construction, $\sigma: H_{*}(P A) \rightarrow H_{*}(\vec{F}(A))$ is represented by $a \rightarrow[a]$ where $P A$ is the subcomplex of primitive chains.

Definition 1. The Massey Product $\langle u\rangle^{k}$. (Kraines [6]).

Let $A$ be a $D G A$ algebra over $K$. Suppose $a_{1}, \cdots, a_{k-1}$ are given in $A$ such that $a_{1}$ is a cycle (or cocycle) and that

$$
\partial a_{n}=\sum_{r=1}^{n-1}(-1)^{\operatorname{deg} a_{r}} a_{r} a_{n-r} \text { for } n=2, \cdots, k-1 .
$$

Suppose $u$ is represented by $a_{1}$. Then the Massey Product $\langle u\rangle^{k}$ is represented by the cycle

$$
\sum_{r=1}^{k-1}(-1)^{\operatorname{deg} a_{r}} a_{r} \cdot a_{k-r}
$$

Theorem 1. (Kraines, [6]). The operation $\langle u\rangle^{k}$ depends only on the class $\left\{a_{1}\right\} \in H(A)$.

Definition 2. (Gitler, [5]). Suppose that $A$ is an associative $D G A$ algebra. Suppose $x \in H(A)$ is such that $v^{k}=0$. The transpotence $\varphi_{k}(v) \in H(\bar{B}(A))_{\text {IImo }}$ is defined as follows: If $b \in A$ represents $v$ then there exists $M \in A$ such that $\partial M=-b^{k} . \varphi_{k}(v)$ is represented by

$$
(-1)^{w}\left[b^{k-1} \mid b\right]+[M] \text { where } w=(1)^{\operatorname{deg} b^{k-1}}+1 \text {. }
$$




\section{Main Theorem.}

THEOREM 2. Let $C$ be a co-associative DGA coalgebra over $K$ and let $A$ be the dual associative $D G A$ algebra over $K$. Suppose $H(A ; K)$ and $H(\bar{B}(A) ; K)$ are free and of finite type over $K$. Let $v$ in $H(A)$ and $v$ in $H(\bar{F}(C) ; K)$ be such that the Kronecker index $\langle\sigma(v), u\rangle$ is 1 . Then $\varphi_{k}(v)$ is defined and is not zero in $H(\bar{B}(A) ; K)$ if and only if $\langle u\rangle^{k}$ is defined and not zero in $H(\bar{F}(C) ; K)$. In this case

$$
\left\langle\varphi_{k}(v),\langle u\rangle^{k}\right\rangle=1 \text {. }
$$

In order to prove this theorem we shall consider the EilenbergMoore Spectral Sequences with

$E^{2}=\operatorname{Cotor}^{H(\bar{B}(A) ; K)}(K, K)$

$E^{r} \Rightarrow E^{\circ} H(\bar{F}(\bar{B}(A)) ; K) \approx H(A ; K)$ as algebras, and dually,

$\left(E^{\prime}\right)^{2}=\operatorname{Tor}^{H\left(\bar{F}^{\prime}(C) ; K\right)}(K, K)$

$\left(E^{\prime}\right)^{r} \Rightarrow E^{\circ} H(B(\bar{F}(\bar{C}) ; K) \approx H(C ; K)$ as coalgebras.

We also note that the Kronecker Index $\langle\rangle:, C \otimes A \rightarrow K$ induces a pairing

$$
\langle,\rangle: \bar{F}(C) \otimes \bar{B}(A) \rightarrow K
$$

Lemma 1. Let $b \in A$ represent $v \in H(A)$. Suppose $v^{k}=0$. Then

$$
d_{k}\left[\varphi_{k}(v)\right]=[\sigma b]^{k} \text { in } E^{k} .
$$

Proof. Let

$$
V=\sum_{i=1}^{k-1} P(i)\left[\left[b^{i} \mid b\right]\right]([[b]])^{k-i-1} \text { where } P(i)=(-1)^{\operatorname{deg} b^{i}+1}
$$

and the outside bars refer to the Cobar Construction and the inside bars refer to the Bar Construction.

Taking $\partial V$ gives a telescoping series and so

$$
\partial V=[\sigma b]^{k}+(-1)^{w}\left[\sigma\left(b^{k}\right)\right] \text {. Here }(-1)^{w}=P(k-1) .
$$

In $E^{1}, V$ represents the class $(-1)^{w}\left[\left[b^{k-1} \mid b\right]\right]+[[M]]=\left[\varphi_{k}(v)\right]$.

The Lemma follows from the definition of a spectral sequence of a bi complex.

Lemma 2. Let $a \in \bar{F}(C)$ represent $u$. Then, by definition,

$$
\gamma_{k}[a]=[a|\cdots(k) \cdots| a] \in \bar{B}(\bar{F}(C)) .
$$

If $\gamma_{k}[a]$ lives to $E^{k-1}$ then $\langle u\rangle^{k}$ is defined and

$$
d_{k}\left(\gamma_{k}[a]\right)=\langle u\rangle^{k} \text { in }\left(E^{\prime}\right)^{k} \text {. }
$$


Proof. We first make an observation: Suppose $\langle u\rangle^{t}$ is defined. Let $\left(a_{i}\right)$ be a defining system for $\langle u\rangle^{t}$. Let

$$
W=\sum_{r=2}^{t} \sum_{i_{1}+\cdots+i_{r}=t}\left[a_{i_{1}}|\cdots| a_{i_{r}}\right] \in \bar{B}(\bar{F}(C)) .
$$

Then

$$
\partial W=\sum_{i=1}^{t-1}(-1)^{\operatorname{deg} a_{i}+1}\left[a_{i} a_{t-i}\right] .
$$

Now to prove Lemma 2, we use induction on $k$. Suppose the lemma is true for $k-1$. Suppose $\gamma_{k}[a]$ lives to $E_{k-1}$. Since $E$ is a spectral sequence of $D G A$ coalgebras, and $d_{k-1}\left(\gamma_{k}[a]\right)=0$, we have

$$
\Delta d_{k-1} \gamma_{k}[\alpha]=d_{k-1}^{\otimes} \Delta \gamma_{k}[\alpha]=d_{k-1}^{\otimes} \sum_{i=0}^{k} \gamma_{i}[\alpha] \otimes \gamma_{k-i}[\alpha]=0
$$

where $d^{\otimes}$ is the differential in $E^{\prime} \otimes E^{\prime}$. That is, in particular when $i=k-1$ in the above, we see

$$
d_{k-1} \gamma_{k-1}[a] \otimes[a]=0 \text { so } d_{k-1} \gamma_{k-1}[a]=0 .
$$

Now by inductive hypothesis, $\langle u\rangle^{k-1}$ is defined so there is a defining system $\left(a_{1}, \cdots, a_{k-1}\right)$ for $\langle u\rangle^{k-1}$ and a cochain $a_{k}$ such that

$$
\delta a_{k}=\sum_{i=2}^{k-2}(-1)^{\operatorname{deg} a_{i-1}} a_{i-1} a_{k-i}
$$

since $\langle u\rangle^{k-1}=d_{k-1} \gamma_{k-1}[a]=0$.

The observation at the beginning of this lemma shows that

$$
d_{k} \gamma_{k}[a]=\langle u\rangle^{k} \text {. }
$$

We now give the proof of Theorem 2: Hence

Assume $\varphi_{k}(v)$ is defined and nonzero. We are assuming $=1=\langle\sigma v, u\rangle$.

$$
\begin{aligned}
1 & =\langle\sigma v, u\rangle=\langle\sigma b, a\rangle=\left\langle[\sigma b]^{k}, \gamma_{k}[a]\right\rangle=\left\langle d_{k} \varphi_{k}(v), \gamma_{k}[a]\right\rangle \\
& =\left\langle\varphi_{k_{k}}(v), d_{k} \gamma_{k}[a]\right\rangle=\left\langle\varphi_{k}(v),\langle u\rangle^{k}\right\rangle
\end{aligned}
$$

by the duality of the two spectral sequences and Lemma 2.

It remains to be shown that if $\langle u\rangle^{k}$ is defined and nonzero, then so is $\varphi_{k}(v)$. Consider the map

$A \rightarrow \bar{F}(\bar{B}(A))$ defined by

$b \rightarrow[[b]]$.

This map is homotopy multiplicative (in fact is a $S H M$ map) and is an equivalence. Hence $\left[\left[b^{k}\right]\right]$ differs from $[\sigma b]^{k}$ by a boundary. But $[\sigma b]^{k}=[\sigma b|\cdots(k) \cdots| \sigma b]$ is dual to $\gamma_{k}[a]=[a|\cdots(k) \cdots| a]$ in $\bar{B} \bar{F}(C)$, and so $d_{k} \gamma_{k}[a]=\langle u\rangle^{k}$ is not zero in $E^{k}$ (Lemma 2) and so does not 
survive to $E^{\infty}$, i.e., represents 0 in $E^{\infty}$. The dual element $[\sigma b]^{k}$ represents 0 in $E^{\infty}$, i.e., $\left[\left[b^{k}\right]\right] \sim[\sigma b]^{k} \sim 0$. Therefore $b^{k} \sim 0$ and so $\varphi_{k}(v)$ is defined.

We wish to mention two applications:

$\mathrm{Al}$ : Let $K=Z_{p}$ and let $X$ be a $K(\pi, n)$ space ( $p$ an odd prime). Let $C=C^{*}\left(X ; Z_{p}\right)$ and $A=C_{*}\left(X ; Z_{p}\right)$ be cochain and chain complexes for $X$ of finite type. In the notation of Cartain, $A=A_{*}\left(\pi, n ; Z_{p}\right)$ ([2]). Cartan proved that $\left\langle\varphi_{p}(v), \beta P^{m}(u)\right\rangle=\langle\sigma v, u\rangle$. Now by Theorem 2, if

$$
\langle\sigma v, u\rangle=1
$$

then $\left\langle\varphi_{p}(v),\langle u\rangle^{p}\right\rangle=1$. Hence $\left\langle\varphi_{p}(v), \beta P^{m} u+\langle u\rangle^{p}\right\rangle=0$. By Lemma 18 ([5]), $\langle u\rangle^{p}=c \beta P^{m} u$. This gives an easy proof of the fact that $c=-1$. (Compare Theorem 19 [5]).

A2: Now let $x=C P^{k-1}$. Then in $H^{*}\left(C P^{k-1} ; Z\right)=P(v)_{l\left(v^{k}\right)}$ we have $v^{k}=0$. Then $\varphi_{l k}(v)$ is defined in $H^{*}\left(\Omega C P^{k-1} ; Z\right)$ and by the Theorem 2, so is $\langle u\rangle^{k}$ in $H_{3 k-2}\left(\Omega C P^{k-1} ; Z\right)$ where $u \in H_{2}\left(\Omega C P^{k-1}, Z\right)$ and $\left\langle\varphi(v),\langle u\rangle^{k}\right\rangle=1$. This gives another proof of the results of Stasheff ([7]).

\section{REFERENCES}

1. J. F. Adams, On the Cobar Construction, Colloquie de Topologie Algébraique, Louvain 1955.

2. H. Cartan et al, Alqébres d'Eilenberq-MacLane et homotopie, Séminaire Henri Cartan, 1954-55. Secrétariat mathématique, Paris, 1956.

3. A. Clark, Homotopy commutativity and the Moore spectral Sequence, Pacific J. Math., 15 (1965), 65-74.

4. B. Drachman, A Diagonal Map for the Cobar Construction Boletín de la Sociedad Matemática Mexicana, 1967.

5. S. Gitler, Espacios Fibrados por H-Espacios. Boletin de la Sociedad Matemática Mexicana, 1963.

6. D. Kraines, Massey higher products. Trans. Amer. Math. Soc., 124 no. 3, (1966). 431-449.

7. J. Stasheff, Homotopy associativity of H-spaces II. Trans. Amer. Math. Soc., 108 no. 2. (1963). 293-312.

Received September 28, 1970.

Michigan State UNIVERSity

AND

AARHUS UNIVERSITY 



\section{PACIFIC JOURNAL OF MATHEMATICS}

\section{EDITORS}

H. SAMELSON

Stanford University

Stanford, California 94305

C. R. HobBY

University of Washington

Seattle, Washington 98105
J. DugundjI

Department of Mathematics

University of Southern California

Los Angeles, California 90007

RICHARD ARENS

University of California

Los Angeles, California 90024

\section{ASSOCIATE EDITORS}

E. F. BECKENBACH

B. H. NeUMaNN

F. WOLF

K. YoshidA

\section{SUPPORTING INSTITUTIONS}

UNIVERSITY OF BRITISH COLUMBIA

CALIFORNIA INSTITUTE OF TECHNOLOGY

UNIVERSITY OF CALIFORNIA

MONTANA STATE UNIVERSITY

UNIVERSITY OF NEVADA

NEW MEXICO STATE UNIVERSITY

OREGON STATE UNIVERSITY

UNIVERSITY OF OREGON

OSAKA UNIVERSITY
UNIVERSITY OF SOUTHERN CALIFORNIA STANFORD UNIVERSITY

UNIVERSITY OF TOKYO

UNIVERSITY OF UTAH

WASHINGTON STATE UNIVERSITY UNIVERSITY OF WASHINGTON

$\stackrel{*}{*} \stackrel{*}{*} \stackrel{*}{*}$ AMERICAN MATHEMATICAL SOCIETY
NAVAL WEAPONS CENTER 


\section{Pacific Journal of Mathematics}

Vol. 39 , No. 1

May, 1971

Charles A. Akemann, A Gelfand representation theory for $C^{*}$-algebras ....

Sorrell Berman, Spectral theory for a first-order symmetric system of

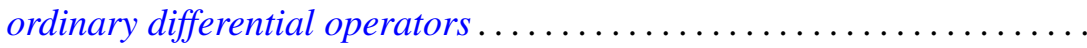

Robert L. Bernhardt, III, On splitting in hereditary torsion theories ........

J. L. Brenner, Geršgorin theorems, regularity theorems, and bounds for determinants of partitioned matrices. II. Some determinantal identities ..........................................

Robert Morgan Brooks, On representing $F^{*}$-algebras .............. 51

Lawrence Gerald Brown, Extensions of topological groups........... 71

Arnold Barry Calica, Reversible homeomorphisms of the real line ........ 79

J. T. Chambers and Shinnosuke Oharu, Semi-groups of local Lipschitzians in

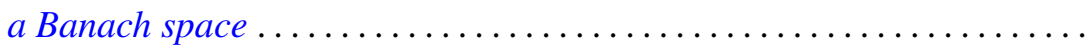

Thomas J. Cheatham, Finite dimensional torsion free rings .............

Byron C. Drachman and David Paul Kraines, A duality between

transpotence elements and Massey products ...................

Richard D. Duncan, Integral representation of excessive functions of a

Markov process ......................................

George A. Elliott, An extension of some results of Takesaki in the reduction

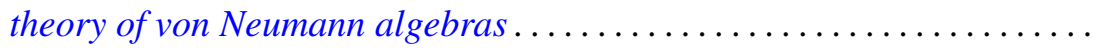

Peter C. Fishburn and Joel Spencer, Directed graphs as unions of partial

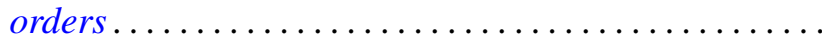

Howard Edwin Gorman, Zero divisors in differential rings ...

Maurice Heins, A note on the Löwner differential equations...

Louis Melvin Herman, Semi-orthogonality in Rickart rings. .

David Jacobson and Kenneth S. Williams, On the solution of linear G.C.D.

equations

Michael Joseph Kallaher, On rank 3 projective planes ... . .

Donald Paul Minassian, On solvable $O^{*}$-groups ...........

Nils Øvrelid, Generators of the maximal ideals of $A(\bar{D})$

Mohan S. Putcha and Julian Weissglass, A semilattice decomposition into

semigroups having at most one idempotent ............

Robert Raphael, Rings of quotients and $\pi$-regularity ....

J. A. Siddiqi, Infinite matrices summing every almost periodic sequence. .

Raymond Earl Smithson, Uniform convergence for multifunctions ...

Thomas Paul Whaley, Mulitplicity type and congruence relations in

universal algebras...

Roger Allen Wiegand, Globalization theorems for locally finitely generated modules... 